\title{
THE IMPACT OF DEUTERATION ON THE INFRARED SPECTRA OF INTERSTELLAR POLYCYCLIC AROMATIC HYDROCARBONS
}

\author{
Douglas M. Hudgins, ${ }^{1,2}$ C. W. Bauschlicher, JR., ${ }^{3}$ and Scott A. Sandford ${ }^{1}$ \\ Received 2004 May 11; accepted 2004 June 30
}

\begin{abstract}
In this paper, we present the results of a computational study of the effects of a low absolute level of deuteration $(\sim 5 \%)$ on the observed mid-IR spectrum of the interstellar polycyclic aromatic hydrocarbon (PAH) population. The analyses are based on a global average spectrum, including all the possible singly deuterated isomers of an ensemble of partially superhydrogenated PAH species (i.e., $\mathrm{H}_{n}$-PAHs) in both neutral and cationic forms. The distinctive spectroscopic properties considered in this paper include three classes of D-involved vibrational modes: (1) $\mathrm{C}-\mathrm{D}$ in-plane bending features in the 9.5-12 $\mu$ m region, (2) $\mathrm{C}-\mathrm{D}$ out-of-plane bending features in the 14.5-17.9 $\mu \mathrm{m}$ region, and (3) aromatic and aliphatic $\mathrm{C}-\mathrm{D}$ stretching features in the 4.4-4.7 $\mu \mathrm{m}$ region. In addition, D substitution also has the potential to alter the appearance of the $\mathrm{C}-\mathrm{H}$ out-of-plane bending features in the 11-14 $\mu \mathrm{m}$ region by disrupting the adjacency of the $\mathrm{H}$ atoms on the PAH edge rings. The spectroscopic impacts of a low level of deuteration in the interstellar PAH population are considered, and their implications for the detailed appearance of the astronomical mid-IR PAH emission spectrum is explored. Overall, the results of this study indicate that the $\mathrm{C}-\mathrm{D}$ stretching features offer the greatest prospects for the detection of deuterated interstellar PAHs. Since these features are effectively obscured by telluric $\mathrm{CO}_{2}$ absorptions, the information presented herein should be useful in the planning and design of future space-based IR observing platforms.
\end{abstract}

Subject headings: astrochemistry — infrared: ISM — ISM: evolution — ISM: molecules molecular processes

Online material: color figures

\section{INTRODUCTION}

Studies of the abundance and distribution of the different isotopic variants of the atoms and molecules in the cosmos can provide valuable insight into the physical and chemical processes that shape our universe. Among the most valuable in this regard are hydrogen $\left({ }^{1} \mathrm{H}\right)$ and its heavy isotope deuterium $\left({ }^{2} \mathrm{H}\right.$ or, more commonly, D), since measurements of the relative distribution of these two isotopes among the various interstellar molecular materials can provide valuable insight into interstellar chemical processes. Of particular interest in this respect is the recent tentative detection of two infrared emission bands at 4.4 and $4.65 \mu \mathrm{m}$ in interstellar spectra attributed to $\mathrm{C}-\mathrm{D}$ stretching vibrations of polycyclic aromatic hydrocarbons (PAHs) carrying one or more D atoms (Peeters et al. 2004). These observations highlight the important insight into interstellar PAH chemistry that dPAHs-PAHs on which one or more of the peripheral $\mathrm{H}$ atoms have been replaced by a $\mathrm{D}$ atom - might one day provide and underscore the need for a better understanding of the infrared spectral properties of those species. Given the low overall cosmic abundance of $\mathrm{D}\left([\mathrm{D}] /[\mathrm{H}] \sim 1 \times 10^{-5}\right.$; Vidal-Madjar et al. 1998), it is likely that singly deuterated forms of PAHs, as opposed to PAHs carrying multiple D atoms, have the greatest impact on the astrophysical problem. The work reported in this paper was undertaken to explore the spectroscopic effects of substituting a $\mathrm{D}$ atom for one of the $\mathrm{H}$ atoms on the periphery of a PAH.

\footnotetext{
${ }^{1}$ Astrophysics Branch, MS 245-6, NASA Ames Research Center, Moffett Field, CA 94035.

${ }^{2}$ Corresponding author; dhudgins@mail.arc.nasa.gov.

3 Space Technology Division, MS 230-3, NASA Ames Research Center, Moffett Field, CA 94035.
}

The $\mathrm{D}$ we find around us today arose from nucleosynthesis in the epoch immediately following the big bang (Reeves et al. 1973). The resultant primordial D/H ratio - ostensibly that observed at cosmological distances-reflects the conditions and processes that characterized the earliest moments of our universe (see, e.g., Epstein et al. 1976; Tytler et al. 1996; Jedamzik \& Fuller 1997; O'Meara et al. 2001). Moreover, since the primordial D is, on balance, consumed in stellar nucleosynthesis, the "local" $\mathrm{D} / \mathrm{H}$ ratio of our own and other nearby galaxies probes the stellar history and evolution of those systems (Pagel 1997). The astrophysical importance of D is not, however, limited solely to its cosmological significance and its value as a tracer of galactic and stellar physics. On the contrary, the occurrence of $\mathrm{D}$ in interstellar molecules also provides direct insight into the physical and chemical conditions throughout the interstellar medium (ISM).

In general, chemical reactions are insensitive to the isotopic masses of the atomic species involved. However, at the extremely low kinetic temperatures that characterize many interstellar environments $(T<100 \mathrm{~K})$, the small absolute masses of $\mathrm{H}$ and $\mathrm{D}$ atoms coupled with their large relative mass difference $\left(m_{\mathrm{D}} / m_{\mathrm{H}} \sim 2\right)$ can lead to large discrepancies in the chemistries of these two species. Under those conditions, the difference in zero-point vibrational energy favors the preferential survival of chemical bonds involving D atoms over those involving $\mathrm{H}$ atoms and often results in the production of deuterated molecular species with relative abundances $([X \mathrm{D}] /[X \mathrm{H}])$ far in excess of the cosmic (atomic) D/H ratio (Geiss \& Reeves 1981; Dalgarno \& Lepp 1984; Tielens 1997). Indeed, the importance of D enrichment in gas-phase interstellar chemistry is well established observationally. Radio-frequency observations of the rotational transitions of the wide array of simple gas-phase interstellar molecules found in cold, dark clouds have shown 
that many of these species exhibit high degrees of D enrichment (see, e.g., Mauersberger et al. 1988; Jacq et al. 1990; Roueff et al. 2000; Turner 2001). Furthermore, the degree of $\mathrm{D}$ enrichment and the pattern of $\mathrm{D}$ substitution are unique to each molecular species and are a direct consequence of the chemical pathway(s) that gave rise to that species (Sandford et al. 2001; Sandford 2002). Consequently, we can learn a great deal about interstellar chemistry by studying the distribution of $\mathrm{D}$ in interstellar molecules.

The presence of deuterated species among the interstellar PAH population, the photoproducts of mixed molecular ice mantles, and other interstellar organics have also been the subject of theoretical (Tielens 1983, 1992, 1997; Millar et al. 1989, 2000; Aikawa \& Herbst 1999) and experimental study. The experimental work has considered both studies of laboratory analogs (see, e.g., Bernstein et al. 1995, 2000; Sandford et al. 2000, 2001) and analyses of meteoritic materials (Kerridge \& Chang 1985; Zinner 1997). Regarding the latter, D enrichments are commonly found in primitive solar system materials, such as carbonaceous chondrites, which contain both circumstellar and interstellar materials that survived the transition from dense cloud to planetary surfaces (Bernatowicz \& Zinner 1997). The excess D in meteorites is largely carried by a variety of organic species, of which aromatics appear to be the most significant (Kerridge et al. 1987; Cronin et al. 1993; Messenger 2000). The combined theoretical and experimental work strongly suggests that interstellar ices and organics, especially PAHs and related aromatic systems, should be significantly enriched in D in many interstellar environments.

Nevertheless, despite the abundance of interstellar PAHs as inferred from the ubiquity of their emission features in space and the mounting evidence that dPAHs should constitute significant carriers of D (Sandford et al. 2001), these species have received little attention in terms of telescopic study. This is due both to the comparative weakness of their expected $\mathrm{C}-\mathrm{D}$ bending and stretching features (even in D-enriched materials the absolute D content is quite low) and to the difficulty of making such observations from the ground due to spectral interference from telluric $\mathrm{CO}_{2}$. As a result, while extensive laboratory and observational work has been done on the spectroscopy of PAHs over the last two decades, relatively little consideration has been given to the impact of deuteration on their emission spectra. That is the purpose of the work reported here.

This paper is organized as follows. In $\S 2$ we briefly review previous experimental and theoretical studies relevant to the production and abundance of dPAHs in interstellar environments. In $\S 3$ we present the results of our theoretical analyses of the infrared spectroscopic properties of singly deuterated PAHs, with subsections devoted to the features arising from each class of fundamental $\mathrm{C}-\mathrm{D}$ vibrational mode. We also discuss the likely impact of the presence of a $\mathrm{D}$ atom on the associated $\mathrm{C}-\mathrm{H}$ features of the PAH.

\section{DEUTERATED INTERSTELLAR PAHs}

The majority of the interstellar chemical processes that yield high D fractionations - e.g., gas-phase ion-molecule reactions (Geiss \& Reeves 1981), reactions on grain surfaces (Tielens 1983), and isotopic exchange during the irradiation of interstellar ices (Sandford et al. 2000) - do so only at low temperatures $(T<50 \mathrm{~K})$. These processes are therefore restricted to the environments of dense molecular clouds. For a few classes of interstellar molecules, such as PAHs, there exists an additional D-enrichment mechanism that does not require low tem- peratures, namely unimolecular photodissociation (Allamandola et al. 1989). While all four of these distinct astrochemical processes can produce dPAHs, each is expected to produce a dPAH population with a distinctive pattern of $\mathrm{D}$ substitution, both within a given $\mathrm{PAH}$ species and between different PAH species (Sandford et al. 2001).

The most common and well-studied avenue for producing $\mathrm{D}$ enrichments in interstellar molecular species is through ion/molecule reactions occurring in the gas phase at the low temperatures $(T<50 \mathrm{~K})$ characteristic of dense interstellar molecular clouds. The preferential incorporation of $\mathrm{D}$ into molecules occurs as a result of the slightly lower zero-point energy (and, hence, slightly greater stability) of the $\mathrm{C}-\mathrm{D}$ bond compared to a C-H bond (Dalgarno \& Lepp 1984; Herbst et al. 1987; Millar et al. 2000; Tielens 1997). Observational evidence that $D$ fractionation occurs in the ISM via this mechanism is provided by radio-wavelength studies of a wide variety of simple molecules found in dense molecular clouds, many of which show moderate to high degrees of D enrichment (Mauersberger et al. 1988; Jacq et al. 1990; Roueff et al. 2000; Turner 2001). Gas-phase ion/molecule reactions analogous to these are also capable of producing D enrichments in the PAH populations found in these regions (Tielens 1997). In the case of PAHs, larger PAHs are initially preferentially deuterated by virtue of the fact that they have more $\mathrm{H}$ atoms and, consequently, more potential sites for D substitution. However, ultimately all PAHs in the gas phase should show increased D/H ratios as they approach a steady state equilibrium. Since PAHs are considerably more stable and abundant in the ISM than many of the smaller species that show D enrichments $\left(\mathrm{PAH} / \mathrm{H}_{2} \sim\right.$ $10^{-6}$, compared to, e.g., $\mathrm{HCN} / \mathrm{H}_{2} \sim 10^{-8}$ ), and since PAHs carry more $\mathrm{H}$ atoms per molecule than simpler molecules, PAHs are likely to represent the largest reservoir of materials enriched in $\mathrm{D}$ by ion-molecule reactions.

Ion-molecule reactions in the gas phase represent only one possible source of D fractionation in the dense cloud environment. At the temperatures typical of dense interstellar clouds $(T<50 \mathrm{~K})$, most volatile species condense out of the gas phase onto icy grain mantles (Sandford \& Allamandola 1993), where they can participate in gas-grain reactions on the grain surfaces (Tielens \& Hagen 1982; d'Hendecourt et al. 1985; Brown \& Charnley 1990; Hasegawa et al. 1992). In environments where $\mathrm{H} / \mathrm{H}_{2}$ is large, surface reactions with $\mathrm{H}$ atoms will dominate and species such as $\mathrm{CH}_{4}, \mathrm{NH}_{3}$, and $\mathrm{H}_{2} \mathrm{O}$ are produced. Such chemistry should produce large D enrichments in grain mantles where reduction is occurring because of the high atomic D fractionation of the accreting gas (Tielens 1983, 1992, 1997; Charnley et al. 1997). However, there is currently no laboratory evidence suggesting that PAHs can become further reduced through simple $\mathrm{H}$-atom exposure on grain surfaces. Thus, this process may leave PAHs largely unaffected.

As noted above, because of the D-rich nature of the molecular volatiles accreting from the gas phase and because of the subsequent gas-grain reactions with $\mathrm{D}$ atoms that occur on their surface, interstellar ice grains are expected to be significantly enriched in D. Under these circumstances, further deuterated species can be synthesized when these ices are processed by UV and cosmic-ray radiation. Laboratory studies of mixed-molecular ices in which some of the original species are $\mathrm{D}$ enriched show this $\mathrm{D}$ incorporation into many of the reaction products (Bernstein et al. 1994, 1995, 2000; Sandford et al. 2000). Unlike the previously described processes, the effect is not to produce a fractionation of $\mathrm{H}$ from $\mathrm{D}$, but instead to propagate previous fractionations into new molecular 
TABLE 1

Comparison of the Characteristic Positions of $\mathrm{C}-\mathrm{H}$ and $\mathrm{C}-$ D Modes in PaHs and PADs

\begin{tabular}{|c|c|c|c|c|}
\hline \multirow[b]{2}{*}{ Mode } & \multicolumn{2}{|c|}{$\mathrm{C}-\mathrm{H}$ BOND } & \multicolumn{2}{|c|}{$\mathrm{C}-\mathrm{D}$ BOND ${ }^{\mathrm{a}}$} \\
\hline & $\lambda(\mu \mathrm{m})$ & $\tilde{\nu}\left(\mathrm{cm}^{-1}\right)$ & $\lambda(\mu \mathrm{m})$ & $\tilde{\nu}\left(\mathrm{cm}^{-1}\right)$ \\
\hline 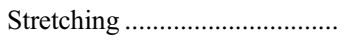 & $3.2-3.3$ & $3100-3000$ & $4.3-4.5$ & $2325-2250$ \\
\hline In-plane bending $\ldots \ldots \ldots \ldots \ldots \ldots \ldots \ldots$ & $7.1-9.1$ & $1400-1100$ & $9.5-12.0$ & $1050-830$ \\
\hline Out-of-plane bending ................. & $10.8-14.0$ & $930-700$ & $13.5-17.9$ & $740-560$ \\
\hline
\end{tabular}

${ }^{\text {a }}$ Bauschlicher et al. (1997).

species. The presence of PAHs in these environments has been indicated by detection of their infrared bands in absorption in the spectra of stars embedded within dense clouds (Sellgren et al. 1995; Brooke et al. 1996, 1999; Chiar et al. 2000; Bregman et al. 2000; Sandford et al. 2004). Laboratory simulations have shown that the irradiation of PAHs in D-enriched ices results in rapid D enrichment of the $\mathrm{PAH}$ population through $\mathrm{H} / \mathrm{D}$ exchange reactions on peripheral aromatic rings and on rings that contain oxygen atoms (Sandford et al. 2000). PAH deuteration by these mechanisms is independent of molecular size but should show specific regiochemical behaviors (Sandford et al. 2001). Further D incorporation in icy grain mantles should also occur through the D-atom addition (as opposed to substitution) reactions that produce D-enriched $\mathrm{H}_{n}$-PAHs, i.e., PAHs that carry excess $\mathrm{H}$ atoms (hereafter we will use the term " $\mathrm{dH}_{n^{-}}$ PAH" to represent an $\mathrm{H}_{n}$-PAH on which one or more of the $\mathrm{H}$ atoms have been replaced by a $\mathrm{D}$ atom). D enrichments generated in this fashion would be expected to correlate with the presence of $\mathrm{H}_{n}$-PAHs, increasing $\mathrm{H} / \mathrm{C}$ ratios, and the presence of aliphatic structures (Sandford et al. 2001).

Finally, in contrast to the foregoing processes that produce D fractionations at very low temperatures, the fractionation effects of unimolecular photodissociation are restricted primarily to the interstellar PAH population but may produce $\mathrm{D}$ enrichments at elevated temperatures (Allamandola et al. 1989; Tielens 1997). PAHs are distinguished by their resistance to photolytic destruction and their resulting persistence in harsh radiation environments where most molecular species are quickly destroyed. However, while PAHs are resistant to photodestruction, they are not immune to photon-mediated modification. In intense, hard UV radiation fields such as those found in planetary nebulae and regions of massive star formation, PAHs may absorb more energy than they can accommodate in their vibrational manifold, leading to bond scission within the molecule. If that break occurs within the carbon skeleton of the $\mathrm{PAH}$, the two resulting fragments are held in close proximity to one another by the remaining carbon skeleton and the bond is likely to reform quickly, returning the excess energy to the vibrational manifold. If, on the other hand, the break occurs at a $\mathrm{C}-\mathrm{H}$ bond on the periphery of the species, the fragments are no longer tethered to one another. In that case, the bond has no opportunity to reform and the excess energy is dispersed largely in the form of the kinetic energy. Because of the zeropoint energy difference between $\mathrm{C}-\mathrm{D}$ and $\mathrm{C}-\mathrm{H}$ bonds, the rate of D loss from interstellar PAHs should be lower than that of $\mathrm{H}$ loss. The resulting PAH radical will subsequently react with ambient H/D atoms, and the process begins again. Because of the modest preference toward $\mathrm{H}$ loss over D loss, repeated processing in this manner should lead to a gradual D enrichment in the PAH population.

Since the vibrational energy capacity of a molecule is proportional to molecular size, the threshold energy needed to exceed this capacity also increases with size. Thus, in a given radiation environment, the foregoing process will preferentially deuterate the smaller species in the interstellar PAH population since the rate of unimolecular photodissociation will increase with decreasing molecular size. Steady state isotopic equilibrium is expected to be reached in this manner in photodissociation regions and, at least for small PAHs, in dense clouds. At equilibrium, the fraction of peripheral $\mathrm{D}$ relative to $\mathrm{H}$ will be 3 times the local gas-phase ratio of $\mathrm{D}$ to $\mathrm{H}\left(10^{-5}\right.$ to $\left.10^{-3}\right)$. Thus, in these interstellar environments, many PAHs having less than $\sim 25 \mathrm{C}$ atoms should have peripheral $\mathrm{D}$ atoms. This process is unique in that the extent of the $\mathrm{D}$ enrichment depends on the size of the PAH and does not require the local environment be cold. D enrichment is expected to be most significant for PAHs in the size range from 10 to $40 \mathrm{C}$ atoms. Enrichment in PAHs having more than $\sim 40 \mathrm{C}$ atoms is not expected because these larger PAHs can accommodate the maximum energy of typical UV photons without photolytic bond cleavage occurring.

Thus, interstellar PAHs are expected to be enriched in D, with the extent, topology, and distribution of that enrichment dependent on the local environment and the PAHs' history (Sandford et al. 2001). This, together with the recent report of the tentative identification of a pair of weak emission features near 4.4 and $4.6 \mu \mathrm{m}$ in the ISO SWS spectrum of Orion bar D5 attributed to dPAHs (Peeters et al. 2004), warrants a closer look at how deuteration might impact the canonical PAH infrared spectrum.

\section{INFRARED SPECTRA OF DEUTERATED PAHS}

$\mathrm{D}$ atoms substituted on the periphery of PAHs participate in the same characteristic types of vibrational motions that $\mathrm{H}$ atoms do. These include $\mathrm{C}-\mathrm{D}$ stretching motions and in-plane and out-of-plane bending vibrations. All of these vibrations are, however, shifted to lower frequencies compared to the analogous $\mathrm{C}-\mathrm{H}$ motions because of the larger mass of the $\mathrm{D}$ atom. Laboratory infrared spectra have been obtained from a number of matrix-isolated perdeuterated PAHs (PADs; i.e., PAHs in which all of the peripheral $\mathrm{H}$ atoms have been replaced by D atoms; Bauschlicher et al. 1997). The characteristic frequencies for the various $\mathrm{C}-\mathrm{D}$ modes in PAHs compared to those of the analogous $\mathrm{C}-\mathrm{H}$ modes are given in Table 1.

However, while the interstellar PAH population is likely to be enriched in D over the canonical interstellar $[\mathrm{D}] /[\mathrm{H}]$ ratio by a combination of the processes discussed in the previous section, they will certainly not be fully deuterated. Indeed, the absolute level of deuteration is unlikely to exceed a few percent. As a result, most of the PAHs in the interstellar population would carry no more than a few D atoms. Assuming a 1:1 correspondence between the intrinsic intensities of corresponding $\mathrm{C}-\mathrm{D}$ and $\mathrm{C}-\mathrm{H}$ vibrational modes, the intensity of 
the resulting interstellar $\mathrm{C}-\mathrm{D}$ features should be no more than a few percent that of the analogous $\mathrm{C}-\mathrm{H}$ bands. This difficulty is further compounded by the fact that two of the three classes of $\mathrm{C}-\mathrm{D}$ vibrational modes (the $4.3-4.5 \mu \mathrm{m} \mathrm{C}-\mathrm{D}$ stretches and the 13.5-17.9 $\mu \mathrm{m} \mathrm{C}-\mathrm{D}$ out-of-plane bends) fall in regions of high atmospheric opacity due to telluric $\mathrm{CO}_{2}$ absorptions and are completely accessible only from space. Detecting the $\mathrm{C}-\mathrm{D}$ vibrational features of deuterated interstellar PAHs therefore constitutes a considerable observational challenge. Nevertheless, with the advent of new instruments and spacebased observational platforms (e.g., ISO, Spitzer, and the James Webb Space Telescope), the high-sensitivity spectroscopic measurements required to detect and study the features of interstellar dPAHs are becoming increasingly feasible.

In an effort to optimize the utility of currently available astronomical data, to provide the basis for future observational studies, and to guide the development of future astronomical infrared instruments and missions, we have undertaken a computational study of the effects of partial deuteration on the observed spectroscopic characteristics of an ensemble of PAHs. The details of the computational approach are discussed in the following section ( $\$ 3.1$ ). Subsequent sections will explore the various distinctive spectroscopic characteristics that would arise in a population of PAHs containing a few percent $\mathrm{D}$ relative to $\mathrm{H}$.

\subsection{Computational Approach}

In recent years, density functional theory has proven to be an excellent method for calculating the vibrational frequencies and intensities of large aromatic molecules (see, e.g., Langhoff 1996). Indeed, these studies have been largely driven by the needs of the astrophysical problem and have provided much valuable insight into the nature of the interstellar PAH population. The computational methodology employed in these studies has been discussed extensively in the literature (see, e.g., Langhoff 1996; Bauschlicher \& Langhoff 1997; Hudgins et al. 2001; Mattioda et al. 2003) and will be summarized only briefly here.

The quantum chemical calculations employed for these studies were performed using the Gaussian 98 computer codes (Frisch et al. 1998). Optimized molecular geometries were calculated and used to compute the harmonic frequencies and infrared intensities for each species using the B3LYP hybrid functional (Stephens et al. 1994; Becke 1993) in conjunction with the 4-31G basis sets (Frisch et al. 1984 and references therein). This level of theory strikes a balance between the competing demands for computational accuracy and computational speed. Calibration calculations (Bauschlicher \& Langhoff 1997) conducted at this level of theory have previously shown that a single scale factor of 0.958 brings the B3LYP/4-31G harmonic frequencies into excellent agreement with experimentally measured fundamental mode positions. Those calibration calculations also show that the computed B3LYP/4-31G intensities are accurate except for the aromatic $\mathrm{C}-\mathrm{H}$ stretches of neutral PAHs, which are overestimated in the calculations by as much as a factor of 2 (interestingly, the intensities calculated for aromatic $\mathrm{C}-\mathrm{H}$ stretches of ionized PAHs and the aliphatic $\mathrm{C}-\mathrm{H}$ stretching fundamentals of both neutral and ionized $\mathrm{H}_{n}-\mathrm{PAHs}$ do not appear to be similarly overestimated; Hudgins et al. 2001). Similar calibration calculations conducted as part of the present study indicate that the calculated intensities of the aromatic $\mathrm{C}-\mathrm{D}$ stretching fundamentals exhibit similar intensity behaviors at this level of theory, as do the $\mathrm{C}-\mathrm{H}$ stretching fundamentals; i.e., the theory provides good intensity results for all

<smiles>CC1(C)C=CC2=Cc3cc4c(cc3C2(C)C)=c2ccc3c1c2C(=CC=4)C=CC=3</smiles><smiles>C[C@H]1c2ccccc2C(C)(C)c2cc3ccc4cccc5ccc2c3c4c51</smiles><smiles></smiles><smiles>CC1(C)C=c2ccc3c4cc5ccccc5cc4cc4ccc(c2c43)C1(C)C</smiles><smiles>CC1(C)c2cccc3ccc4cc5cc6ccccc6cc5c(c4c23)C1(C)C</smiles>


FIG. 1.-Structures of the $\mathrm{H}_{2}$-naphthopyrene $\left(\mathrm{C}_{24} \mathrm{H}_{16}\right)$ and $\mathrm{H}_{4}$-naphthopyrene $\left(\mathrm{C}_{24} \mathrm{H}_{18}\right)$ species upon which the average spectra in Figs. $2-5$ are based. See $\S 3.1$ for details of the spectral averaging procedure. The numerals in the first structure illustrate the adjacency classes of the $\mathrm{C}-\mathrm{H}$ groups associated with each of the peripheral aromatic rings (i.e., $1=$ solo, $2=$ duet, $3=$ trio, and $4=$ quartet; see $\S 3.1$.

but the $\mathrm{C}-\mathrm{D}$ stretching vibrations of neutral $\mathrm{dPAHs}$, which may be as much as a factor of 2 too large.

To facilitate comparisons with astronomical infrared emission spectra, synthetic representations of the computational results have been prepared in which each peak is assigned a nominal $30 \mathrm{~cm}^{-1}$ FWHM, a width that is characteristic of the natural line width of a PAH emitting under interstellar conditions. The utility of such spectra for drawing insights into the observed interstellar spectra is well established in the literature (Allamandola et al. 1999; Hudgins \& Allamandola 1999a, 1999b; Van Kerckhoven et al. 2000; Hony et al. 2001; Peeters et al. 2002). In the course of this work, we first considered deuteration of a series of partially hydrogenated derivatives of the PAH naphtho[2, 3; a]pyrene (hereafter, naphthopyrene). The structures of the species studied are shown in Figure 1. The $\mathrm{H}_{2}$ - and $\mathrm{H}_{4}$-naphthopyrene species were chosen as the principal model compounds for a variety of reasons. First, like the interstellar PAH population, they contain both aromatic as well as aliphatic $\mathrm{C}-\mathrm{H}$ groups. Second, all four of the different aromatic $\mathrm{C}-\mathrm{H}$ adjacency classes commonly reflected in the interstellar 11-14 $\mu \mathrm{m}$ emission (Hony et at. 2001) are represented in the basic structure. The exact position of the PAH features that fall in this region are sensitive to the number of neighboring $\mathrm{C}-\mathrm{H}$ groups on each peripheral aromatic ring (e.g., Bellamy 1958) - termed the "adjacency" of the $\mathrm{C}-\mathrm{H}$ groups. The designation "solo" is used to indicate an aromatic $\mathrm{C}-\mathrm{H}$ group with no immediately adjacent $\mathrm{C}-\mathrm{H}$ groups. The designation "duet" indicates two $\mathrm{C}-\mathrm{H}$ groups adjacent to one another. Likewise, "trio" indicates three adjacent peripheral $\mathrm{C}-\mathrm{H}$ groups and "quartet" indicates four adjacent $\mathrm{C}-\mathrm{H}$ 


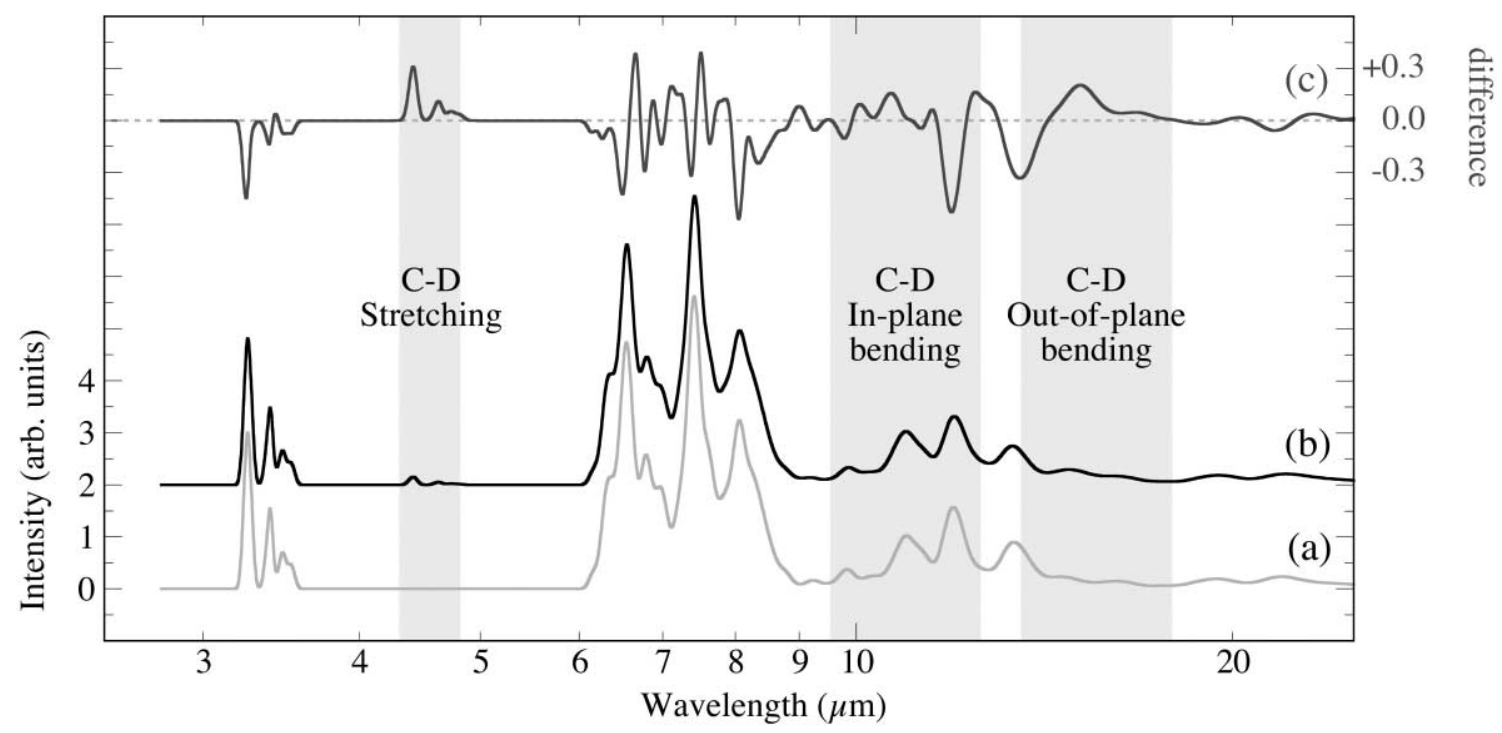

FIG. 2.-Comparison of $(a)$ the average spectrum of the unsubstituted $\mathrm{H}_{n}$-naphthopyrene species shown in Fig. 1 to $(b)$ that of the average of all the possible singly deuterated isomers of those species across the mid-IR. The averages include both neutral and singly ionized states of each species. Trace $c$ shows the difference between traces $a$ and $b$. The characteristic wavelength regimes for the various D-involved vibrational modes of dPAHs are shaded. [See the electronic edition of the Journal for a color version of this figure.]

groups. This concept is illustrated in the first $\mathrm{H}_{2}$-naphthopyrene structure of Figure 1, where the peripheral adjacency class of the $\mathrm{C}-\mathrm{H}$ group(s) on each aromatic ring is indicated by a numeral within that ring (i.e., $1=$ solo, $2=$ duet, $3=$ trio, and $4=$ quartet). Finally, with 16 possible sites, substitution of a single $\mathrm{D}$ atom in the molecule corresponds to a $\mathrm{D}$ content of $6.7 \%([\mathrm{D}] /[\mathrm{H}]=1 / 15)$, a comfortable upper limit to the level of deuteration in the interstellar population. As expected, the spectral changes that accompany substitution of a D atom depend on the site of that substitution and, to some extent, the particular molecule. For example, the spectral changes that occur in molecules that contain aliphatic $\mathrm{C}-\mathrm{H}$ groups are somewhat different than those observed in the purely aromatic parent. In addition, substitution of a D atom at the position of a solo- $\mathrm{CH}$ group is different from substituting one at the position of a duet- $\mathrm{CH}$ group, a trio- $\mathrm{CH}$ group, and so on.

Because the interstellar emission is expected to arise from a mixture of PAHs rather than any one single species, we have averaged the spectra of all the possible singly deuterated isomers of all the naphthopyrene derivatives shown in Figure 1. Again, this would correspond to a $\mathrm{D} / \mathrm{H}$ ratio in the overall population of $6.7 \%$, almost certainly an upper limit to that expected for PAHs in most interstellar environments. Moreover, it is now widely accepted that the features of neutral PAHs dominate the interstellar emission in the 3.3-3.5 and 10-20 $\mu \mathrm{m}$ regions, while those of PAH cations dominate the emission in the 6-9 $\mu \mathrm{m}$ region. Rather than present neutral and cation spectra separately, we have also included both the neutral and cationic forms of the foregoing species in the spectral average. Thus, our averaged spectrum should reflect the spectroscopic effects of the distribution of a few percent D uniformly across the various different structural sites and ionization states available in a generic population of PAHs. Note that we have not scaled the strengths of any of the $\mathrm{C}-\mathrm{D}$ bands, so the relative strength of the $\mathrm{C}-\mathrm{D}$ aromatic stretching feature is probably slightly overestimated. The resulting spectrum is shown in the middle trace of Figure 2 and represents the average of 260 discrete spectra, 130 from neutrals and 130 from cations. The bottom trace in Figure 2 corresponds to the analogous average spectrum of the fully hydrogenated species. The top trace is the difference between these two spectra. A quick inspection of the figure shows that a number of differences do arise from D-atom substitution. However, with the notable exception of the $\mathrm{C}-\mathrm{D}$ stretching features in the 4.4$4.6 \mu \mathrm{m}$ region, the changes do not occur in "clean regions" of the spectrum, but instead suffer from varying degrees of spectral confusion with other vibrational bands. In the following sections we will explore these differences in more detail.

\subsection{The $\mathrm{C}-\mathrm{D}$ In-Plane Bending Region $\left(9.5-12 \mu \mathrm{m} ; 1050-830 \mathrm{~cm}^{-1}\right)$}

It is now well established that the $\mathrm{C}-\mathrm{H}$ in-plane bending modes of PAH cations are strongly enhanced relative to their neutral counterparts and, as a result, dominate the prominent 7.7 and $8.6 \mu \mathrm{m}$ interstellar emission bands. The analogous features of dPAHs, which should fall at somewhat longer wavelengths $(9.5-12 \mu \mathrm{m})$, might therefore seem to offer excellent potential as targets for an interstellar search. However, this potential is not borne out by our calculations. Figure 3 shows an expanded plot of the spectrum in Figure 2 in this spectral region. The canonical $\mathrm{C}-\mathrm{D}$ in-plane bending region is indicated by a shaded bar on the diagram, as is the shortwavelength end of the $\mathrm{C}-\mathrm{H}$ out-of-plane bending region. Inspection of the figure reveals that the features of unsubstituted PAHs that fall shortward of the classic $\mathrm{C}-\mathrm{H}$ out-of-plane bending features (i.e., $\lambda<11 \mu \mathrm{m}$ ) are every bit as prominent as the strongest expected $\mathrm{C}-\mathrm{D}$ features in that region. The reason for this is twofold. First, although the $\mathrm{C}-\mathrm{H}$-involved features that fall in this region are intrinsically weaker than the $\mathrm{C}-\mathrm{D}$ inplane bending fundamentals, $\mathrm{C}-\mathrm{H}$ groups are far more abundant that $\mathrm{C}-\mathrm{D}$ groups in the population. Second, the prodigious intensities of the $\mathrm{C}-\mathrm{H}$ in-plane bending modes in PAH cations are an indirect consequence of Fermi resonance with the $\mathrm{C}-\mathrm{C}$ stretching modes of the molecule, which are directly enhanced by ionization and whose characteristic energies (1600-1200 $\mathrm{cm}^{-1}$ ) overlap those of the $\mathrm{C}-\mathrm{H}$ in-plane modes (1400-1100 $\mathrm{cm}^{-1}$ ). This is not, however, true for the $\mathrm{C}-\mathrm{D}$ in-plane bending 


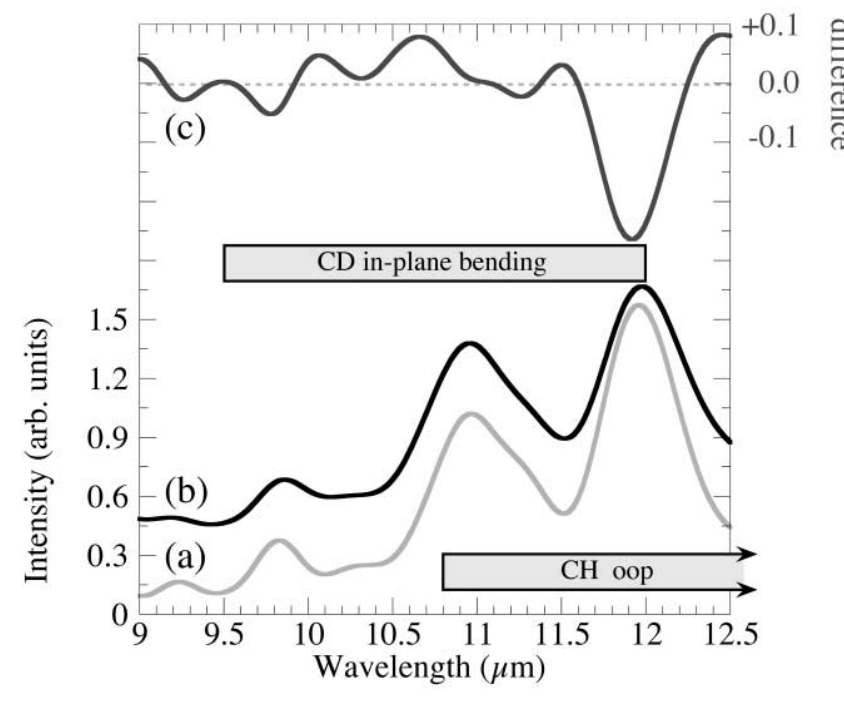

FIg. 3.-Expanded view of the average unsubstituted- and $\mathrm{d}_{1}-\mathrm{H}_{n}-$ naphthopyrene spectra (traces $a$ and $b$, respectively) through the $\mathrm{C}-\mathrm{D}$ in-plane bending region. Weak in the neutral species, these modes are too far removed from the $\mathrm{C}-\mathrm{C}$ stretching modes to experience any significant resonant enhancement in the cations as well (see discussion in § 3.2). Consequently, as shown in the difference spectrum, the result is a weak feature or features in the $10-11 \mu \mathrm{m}$ range that are lost amid an array of features from the unsubstituted species. [See the electronic edition of the Journal for a color version of this figure.]

modes, whose characteristic energies $\left(1050-830 \mathrm{~cm}^{-1}\right)$ do not overlap those of the $\mathrm{C}-\mathrm{C}$ stretching modes and therefore do not experience the same resonant enhancement. As a result, the $\mathrm{C}-\mathrm{D}$ in-plane modes in PAH cations are intrinsically weaker than their corresponding modes in the hydrogenated cations. Together, these two factors - low $\mathrm{C}-\mathrm{D}$ group abundance and lack of resonant enhancement - dictate that any $\mathrm{C}-\mathrm{D}$ in-plane vibrational features of dPAHs that might fall in this region of the spectrum are likely to be no more prominent than the minor features that might naturally be expected in the emission spectra of normal, unsubstituted PAHs. Thus, even if a feature or features were discerned in this region, it would be difficult to establish an unequivocal connection to dPAHs.

\subsection{The C-D Out-of-Plane Bending Modes (13.5-17.9 $\mu \mathrm{m} ; 740-560 \mathrm{~cm}^{-1}$ )}

The $\mathrm{C}-\mathrm{D}$ out-of-plane bending modes that fall in the $13.5-$ $17.9 \mu \mathrm{m}$ region offer another possibility for detecting the spectral signature of interstellar dPAHs. Exhibiting respectable intensities in both neutral and ionized species, these modes are the analogs of the $\mathrm{C}-\mathrm{H}$ out-of-plane bending modes that produce the familiar $11.2 \mu \mathrm{m}$ feature and its accompanying substructure in the 11-14 $\mu \mathrm{m}$ region of the interstellar emission spectrum.

Figure 4 shows an expanded version of the spectra in Figure 2 to facilitate a closer examination of this region of the spectrum. As has been widely discussed in the literature (e.g., Hudgins \& Allamandola 1999b; Hony et al. 2001), the out-ofplane motions of groups of $\mathrm{H}$ atoms lying adjacent to one another on the same peripheral ring of a PAH can couple together. These couplings alter the energy of the vibrations and confer upon each adjacency class its own more-or-less unique, characteristic frequency range. It is important to note that this will not be the case for the D atoms in interstellar dPAHs. First, because of its greater mass and different vibrational frequency,

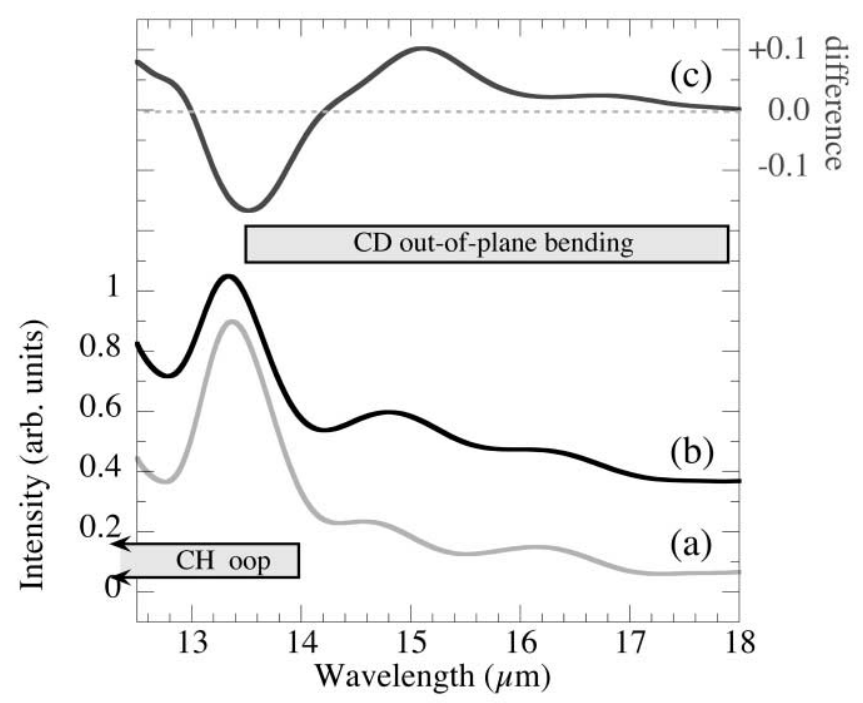

FIG. 4.-Expanded view of the average unsubstituted- and $\mathrm{d}_{1}-\mathrm{H}_{n}-$ naphthopyrene spectra (traces $a$ and $b$, respectively) through the $\mathrm{C}-\mathrm{D}$ out-ofplane bending region. The difference spectrum ( $\operatorname{trace} c$ ) shows that, on average, modest D substitution gives rise to a broad, low contrast plateau between 14.5 and $17 \mu \mathrm{m}$ that blends with the skeletal distortion modes of the unsubstituted species that also fall in this region. [See the electronic edition of the Journal for a color version of this figure.]

the out-of-plane motions of $\mathrm{D}$ atoms do not couple to those of the adjacent $\mathrm{H}$ atoms. Second, the low absolute level of deuteration in the interstellar PAH population makes it highly unlikely that even two D atoms will be adjacent to one another on the edge of a PAH, let alone three or more. Consequently, unlike $\mathrm{H}$ atoms whose adjacency is determined by the edge structure of the carbon skeleton, essentially all the D atoms in the interstellar PAH population will be solo D atoms, regardless of the edge structure of the carbon skeleton to which they reside. Their presence will, however, change the adjacency class of the $\mathrm{H}$ atoms on the same ring.

The presence of $\mathrm{D}$ atoms affects the $13-18 \mu \mathrm{m}$ region of the PAH spectrum in several ways. Inspection of the difference spectrum in the top panel of Figure 4 shows that the presence of $\mathrm{D}$ at the $\mathrm{D} / \mathrm{H} \sim 6.7 \%$ level gives rise to a broad, low-level "solo-CD" plateau extending from about 14.2 to $16.5 \mu \mathrm{m}$ (700 to $600 \mathrm{~cm}^{-1}$ ). This contrasts with the characteristic solo $\mathrm{C}-\mathrm{H}$ out-of-plane bending modes that fall in a much narrower spectral range (10.9-11.4 $\mu \mathrm{m}, \Delta \lambda \sim 0.5 \mu \mathrm{m} ; 920-875 \mathrm{~cm}^{-1}$, $\Delta \nu \sim 45 \mathrm{~cm}^{-1}$ ) and combine to produce a prominent, discrete band. This larger variance is likely attributable to the greater structural variability associated with the solo-CD modes compared to the solo- $\mathrm{CH}$ modes. As mentioned above, the adjacency class of a $\mathrm{C}-\mathrm{H}$ group on the periphery of a PAH is determined by the geometry of the edge structure of the PAH. As a case in point, solo- $\mathrm{CH}$ modes correspond specifically to $\mathrm{C}-\mathrm{H}$ groups that are adjacent to two "bridgehead" $\mathrm{C}$ atoms, $\mathrm{C}$ atoms that are bonded only to other $\mathrm{C}$ atoms in the structures (see Fig. 1). Consequently, the structural nature of solo-CH groups is quite homogeneous; hence the narrow characteristic frequency range associated with these modes. $\mathrm{C}-\mathrm{D}$ groups, on the other hand, are all solo by virtue of the low isotopic abundance of D, not because the structural characteristics of their location on the parent PAH. Thus, for example, although a solo-CD group may occupy a structural solo position as defined above, we may just as likely (if not more so) find a 


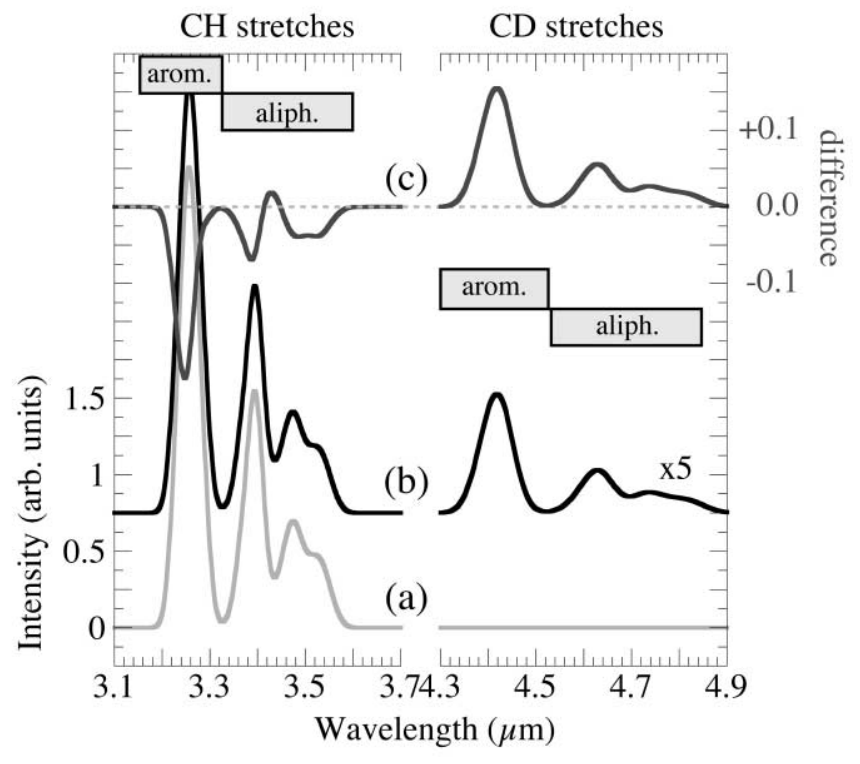

FIG. 5.-Expanded view of the average unsubstituted- and $\mathrm{d}_{1}-\mathrm{H}_{n}-$ naphthopyrene spectra (traces $a$ and $b$, respectively) through the $\mathrm{C}-\mathrm{H}$ and $\mathrm{C}-\mathrm{D}$ stretching regions. Trace $c$ shows the difference between the two spectra. The subregions characteristic of aromatic and aliphatic stretches are indicated by shaded bars. The figure illustrates that the $\mathrm{C}-\mathrm{D}$ stretching modes of dPAHs are expected to produce distinct features that fall in a region of the spectrum free of features from the unsubstituted species. The $\mathrm{C}-\mathrm{D}$ stretching region of trace $b(4.3-4.9 \mu \mathrm{m})$ has been multiplied by a factor of 5 to enhance visibility. [See the electronic edition of the Journal for a color version of this figure.]

solo-CD group occupying one of the positions in what would structurally be classified as a duet, trio, or quartet edge structure if only $\mathrm{H}$ atoms were present. Consequently, the molecular environment experienced by solo-CD groups in the interstellar $\mathrm{PAH}$ population is significantly more heterogeneous than that associated with the corresponding solo- $\mathrm{CH}$ groups. This results in a broader spectral range into which these $\mathrm{C}-\mathrm{D}$ out-of-plane vibrations fall.

As a consequence of this behavior, if the level of $\mathrm{D}$ enrichment in the interstellar PAH population were as high as $\sim 10 \%$, we would expect a broad, low-level plateau with about $10 \%$ the intensity of the $11.2 \mu \mathrm{m}$ band extending over the $\sim 2 \mu \mathrm{m}$ range between 14 and $16 \mu \mathrm{m}$. While not out of the question, the difficulty of this observation is compounded by the facts that there are strong telluric $\mathrm{CO}_{2}$ absorptions in this spectral region, that the background dust continuum in many sources is rising strongly through this spectral region, and that the $\sim 7 \%$ level of deuteration reflected in Figure 4 represents an optimistic upper limit. Thus, as was the case for the $\mathrm{C}-\mathrm{D}$ in-plane bending region discussed above, it will likely be difficult to obtain unambiguous assignments of features in the $\mathrm{C}-\mathrm{D}$ out-of-plane bending region due to dPAHs.

\subsection{The Aromatic $\mathrm{C}-\mathrm{D}$ Stretching Modes $\left(4.3-4.5 \mu \mathrm{m} ; 2320-2220 \mathrm{~cm}^{-1}\right)$}

The final class of dPAH vibration that directly involves motion of the $\mathrm{D}$ atom is stretching of the $\mathrm{C}-\mathrm{D}$ bond. These modes fall in the $4.31-4.51 \mu \mathrm{m}\left(2320-2220 \mathrm{~cm}^{-1}\right)$ region. This wavelength region is obscured by a strong telluric $\mathrm{CO}_{2}$ feature, so detection of such a feature in the interstellar spectrum requires a space-based instrument. An expanded plot of the relevant data from the $\mathrm{C}-\mathrm{H}$ and $\mathrm{C}-\mathrm{D}$ stretching regions is shown in Figure 5.
Figure 5 demonstrates that deuteration at the $\sim 7 \%$ level would give rise to a feature near $4.4 \mu \mathrm{m}\left(2275 \mathrm{~cm}^{-1}\right)$ with approximately $10 \%$ the intensity of the corresponding $3.3 \mu \mathrm{m}$ $\left(3050 \mathrm{~cm}^{-1}\right)$ feature. Unlike the $\mathrm{C}-\mathrm{D}$ in-plane bending modes, these features are not substantially suppressed, on a per bond basis, when compared to the analogous $\mathrm{C}-\mathrm{H}$ modes. Furthermore, unlike the $\mathrm{C}-\mathrm{D}$ out-of-plane bending modes, these modes are not particularly sensitive to peripheral structure. Consequently, rather than smearing out over a wide region, the $\mathrm{C}-\mathrm{D}$ stretching features of different molecular variants pile up to give a single discrete aromatic $\mathrm{C}-\mathrm{D}$ feature, independent of the placement of the D. Finally, there are no features of nondeuterated PAHs or $\mathrm{H}_{n}-\mathrm{PAHs}$ that fall anywhere near this position in the spectrum. Therefore, any PAH feature identified in this region could be unambiguously attributed to deuterated PAHs. Consequently, the $\mathrm{C}-\mathrm{D}$ stretching feature of deuterated PAHs clearly presents the best candidate for providing an identification of these species in the interstellar emission spectrum.

\subsection{The Aliphatic $\mathrm{C}-\mathrm{D}$ Stretching Modes (4.6-4.8 $\mu \mathrm{m} ; 2170-2080 \mathrm{~cm}^{-1}$ )}

The aromatic $\mathrm{C}-\mathrm{H}$ stretching feature observed at $3.3 \mu \mathrm{m}$ in the interstellar PAH emission spectrum is commonly accompanied by secondary features in the $3.4-3.5 \mu \mathrm{m}$ range. These features can be attributed to the stretching of aliphatic $\mathrm{C}-\mathrm{H}$ bonds in side chains (see, e.g., Sandford 1991) or partially reduced PAH species $\left(\mathrm{H}_{n}\right.$-PAHs; Bernstein et al. 1996) and are clearly visible in the model spectrum of the singly deuterated $\mathrm{H}_{n}$-PAHs shown in Figure 5. Recent observations have shown that species such as these are important components of the circumstellar outflows in which the PAH population is initially formed (Goto et al. 2003). Moreover, as discussed above, these species are also a natural consequence of the photochemical processing of PAHs in interstellar ices (Bernstein et al. 1996, $1999,2001)$. Since the same processes that produce $\mathrm{H}_{n}-\mathrm{PAHs}$ under these conditions should also lead to D fractionations (Sandford et al. 2001), it is reasonable to anticipate that aliphatic $\mathrm{C}-\mathrm{D}$ groups will also be produced. In these cases a significant portion of the $\mathrm{D}$ is sited on the peripheral aliphatic rings of the parent PAH (Sandford 2002). As is the case for the $\mathrm{C}-\mathrm{H}$ stretching modes, aliphatic $\mathrm{C}-\mathrm{D}$ stretching features should fall at a slightly longer wavelength than do aromatic $\mathrm{C}-\mathrm{D}$ stretches. These aliphatic $\mathrm{C}-\mathrm{D}$ features are clearly visible in the model spectrum of Figure 5 and do, in fact, fall in the $4.53-4.88 \mu \mathrm{m}\left(2210-2050 \mathrm{~cm}^{-1}\right)$ region longward of the aromatic C-D stretches.

Since both aromatic and aliphatic $\mathrm{C}-\mathrm{D}$ stretching features lie in the relatively uncluttered spectral region near $4.5 \mu \mathrm{m}$, they represent the best candidates for an unambiguous observation of interstellar dPAHs and $\mathrm{dH}_{n}$-PAHs. Because of this importance, to confirm that the results reflected in the model spectrum are generally representative of such species, we have undertaken calculations for several additional monodeuterated $\mathrm{H}_{n}$-PAHs. These included hydrogenated derivatives of the structurally compact PAHs coronene and circumcoronene shown in Figure 6 (24 and $54 \mathrm{C}$ atoms, respectively). The average calculated positions of the $\mathrm{C}-\mathrm{D}$ stretching features for each of these new classes of dPAHs, as well as those for the monodeuterated $\mathrm{H}_{n}$-naphthopyrene species encompassed by the model spectrum, are presented in Table 2 . In addition, the monodeuterated $\mathrm{H}_{n}$-naphthopyrene data are further broken down to compare the average $\mathrm{C}-\mathrm{D}$ stretching positions in the neutral species to those in the corresponding cations. 
<smiles></smiles>

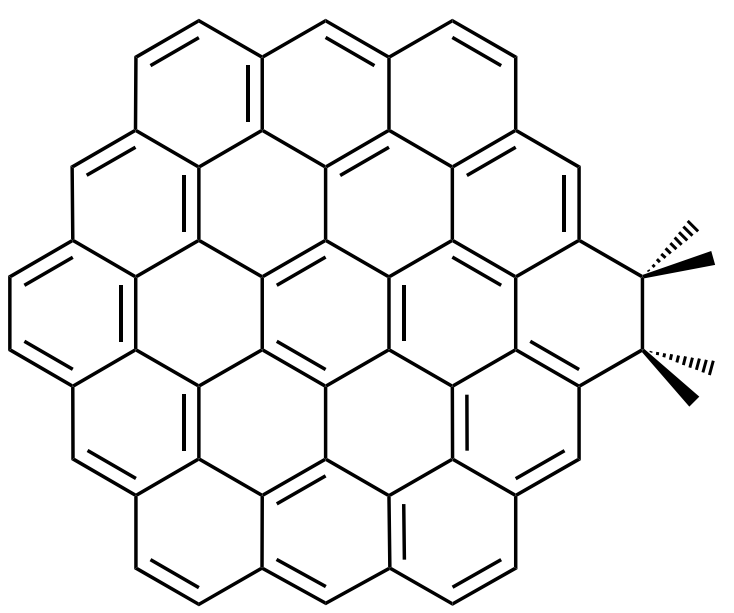

FIG. 6. - Structures of the $\mathrm{H}_{2}$-coronene $\left(\mathrm{C}_{24} \mathrm{H}_{14}\right)$ and $\mathrm{H}_{2}$-circumcoronene $\left(\mathrm{C}_{54} \mathrm{H}_{20}\right)$ species upon which the average data included in Table 2 are based. See $\S 3.1$ for details of the spectral averaging procedure.

Inspection of the data in Table 2 shows that the positions of the aromatic and aliphatic $\mathrm{C}-\mathrm{D}$ stretching features of dPAHs are surprisingly insensitive to molecular size and symmetry and position of $\mathrm{D}$ substitution. Ionization appears to produce a slight blueshift in both classes of bands, although the effect is small $(<0.05 \mu \mathrm{m})$. As is the case for the aromatic $\mathrm{C}-\mathrm{H}$ stretches (Langhoff 1996), the total intensity of the aromatic $\mathrm{C}-\mathrm{D}$ stretching features is suppressed in the cations. It should be noted, however, that this is not the case for the aliphatic $\mathrm{C}-\mathrm{D}$ stretching features. Calibration calculations conducted as part of the present study show that the total intensities of the aliphatic $\mathrm{C}-\mathrm{D}$ stretching modes are, instead, similar in the neutral and cationic species. This striking difference in the ionization effect on the two classes of C-D stretching vibrations is consistent with that reported previously for the analogous $\mathrm{C}-\mathrm{H}$ stretching modes (Hudgins et al. 2001).

The aromatic $\mathrm{C}-\mathrm{D}$ stretching bands fall in a very narrow wavelength range, while the aliphatic values show a somewhat larger spread in values. The origin of the larger range for the aliphatic values arises because they actually fall into two ranges, with average values of 4.625 and $4.757 \mu \mathrm{m}$ (2162 and $2102 \mathrm{~cm}^{-1}$ ). These are due to asymmetric and symmetric $\mathrm{C}-\mathrm{H}$ stretching modes of the $\mathrm{CH}_{2}$ groups, respectively. However, since the natural line width of interstellar species is about $30 \mathrm{~cm}^{-1}(0.066 \mu \mathrm{m}$ at this wavelength), the features due to these two modes will overlap significantly and this small difference may be difficult to observe. Consequently, we believe the $\mathrm{C}-\mathrm{D}$ stretching positions reported here should be generally representative of those that might be observable from the interstellar emission zones, regardless of the ionization balance and the detailed composition of the $\mathrm{PAH} / \mathrm{H}_{n}-\mathrm{PAH}$ population.

\subsection{The Effects of Monodeuteration on the $\mathrm{C}-\mathrm{H}$ Out-of-Plane Bending Modes of PAHs}

So far we have focused on the $\mathrm{C}-\mathrm{D}$ bands, but replacing one $\mathrm{H}$ atom with a $\mathrm{D}$ atom might, in principal, also exert an effect on the $\mathrm{C}-\mathrm{H}$ modes of the molecule. As noted above, because of their different isotopic masses, the vibrational motions of $\mathrm{D}$ atoms do not couple with those of the surrounding $\mathrm{H}$ atoms. Nowhere is this coupling more intimately tied to spectral appearance than for the $\mathrm{C}-\mathrm{H}$ out-of-plane bending modes whose vibrational frequencies are sensitive to the number of adjacent $\mathrm{H}$ atoms (see $\S 3.3$ ). D substitution disrupts the adjacency of the peripheral ring to which it is attached, thereby altering the fundamental vibrational motions of any remaining $\mathrm{H}$ atoms on the ring. The effect of D substitution on the various PAH adjacency classes is illustrated in Figure 7. Substitution of the D atom at a solo- $\mathrm{CH}$ position would eliminate that group entirely (leaving only a solo $\mathrm{CD}$ ). Substitution into a duet $\mathrm{CH}$ on the other hand produces a solo $\mathrm{CH}$ at the expense of the original duet group. Substitution at a trio-CH position produces either a duet-CH group ( $\frac{2}{3}$ probability) or a pair of solo- $\mathrm{CH}$ groups $\left(\frac{1}{3}\right.$ probability). Finally, substitution for a quartet-CH group produces either a trio-CH group or one solo- and one duet-CH group (with equal probability).

Nevertheless, while this substitution can certainly have a profound impact on the spectrum of any particular species, inspection of the 11-14 $\mu \mathrm{m}$ region of Figure 2 shows that these variances wash out when averaged over all the possible substitutional positions in a large dPAH population. Thus, in the absence of a strong bias toward substitution at a particular position, the spectral impact of $\mathrm{D}$ substitution on the $\mathrm{C}-\mathrm{H}$ vibrational modes of the interstellar PAH population would likely be negligible.

\subsection{PAHs with Multiple Deuteration}

In the previous sections we conservatively restricted ourselves to considering PAHs to which only one deuterium atom

TABLE 2

Average C - D Stretching Frequencies for Several Singly Deuterated PaHs

\begin{tabular}{|c|c|c|c|c|}
\hline \multirow[b]{2}{*}{ PAH } & \multicolumn{2}{|c|}{ ARomatic C-D Stretching } & \multicolumn{2}{|c|}{ Aliphatic C-D Stretchinc } \\
\hline & $\lambda(\mu \mathrm{m})$ & $\tilde{\nu}\left(\mathrm{cm}^{-1}\right)$ & $\lambda(\mu \mathrm{m})$ & $\tilde{\nu}\left(\mathrm{cm}^{-1}\right)$ \\
\hline $\mathrm{H}_{n}$-naphthopyrene ${ }^{\mathrm{a}} \ldots \ldots \ldots \ldots$ & 4.427 & 2259 & 4.690 & 2132 \\
\hline 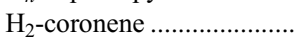 & 4.431 & 2257 & 4.686 & 2134 \\
\hline $\mathrm{H}_{2}$-circumcoronene ........... & 4.435 & 2255 & 4.675 & 2139 \\
\hline $\mathrm{H}_{n}$-naphthopyrene ${ }^{+, \mathrm{a}} \ldots \ldots \ldots$ & 4.404 & 2271 & 4.670 & 2141 \\
\hline
\end{tabular}

NotE.- Species are neutral unless otherwise indicated.

${ }^{a}$ Mixture of $\mathrm{H}_{2}$ - and $\mathrm{H}_{4}$-naphthopyrene isomers from Fig. 1. 
(a)



1 solo- $\mathrm{CH}$

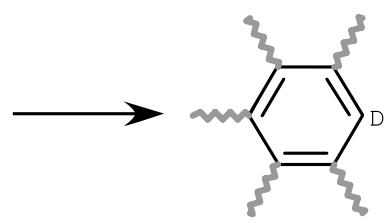

1 solo-CD

(b)<smiles>CCCc1c(C)ccc(C)c1C</smiles>

2 duet- $\mathrm{CH}$

1 solo- $\mathrm{CH}+1$ solo- $\mathrm{CD}$


2 duet- $\mathrm{CH}+1$ solo- $\mathrm{CH}+1$ solo- $\mathrm{CD}$

FIG. 7.- Schematic illustration of the possible effects of substitution of a single D atom on the C- $\mathrm{H}$ adjacency for $(a)$ solo- $\mathrm{CH}$ groups, $(b)$ duet- $\mathrm{CH}$ groups, (c) trio- $\mathrm{CH}$ groups, and $(d)$ quartet- $\mathrm{CH}$ groups. The jagged gray lines indicate bonds to the remaining carbon skeleton of the PAH.

had been added (see $\S 3$ ). However, the recent discoveries of a number of multiply deuterated gas-phase species in the ISM, for example $\mathrm{D}_{2} \mathrm{CO}$ (Loinard et al. 2000), $\mathrm{NHD}_{2}$ and $\mathrm{ND}_{3}$ (Roueff et al. 2000; van der Tak et al. 2002), $\mathrm{CHD}_{2} \mathrm{OH}$ (Parise et al. 2002), and $D_{2} S$ (Vastel et al. 2003), indicate that multiple deuteration is more common than predicted in earlier chemical models. The cause(s) of these larger than expected deuteration levels are not currently fully understood, although gas-phase ion-molecule reactions involving $\mathrm{D}_{2} \mathrm{H}^{+}$and $\mathrm{D}_{3}{ }^{+}$(Vastel et al. 2004) and gas-grain reactions in regions of enhanced $\mathrm{D} / \mathrm{H}$ (Parise et al. 2002) may well play a role.

In any event, interstellar PAHs are directly or indirectly susceptible to deuteration by these very same processes (Sandford et al. 2001), thereby raising the possibility that some significant fraction of the PAHs in the interstellar population might carry multiple D atoms. The presence of multiply deuterated PAHs will produce some changes in the predicted spectrum of the overall $\mathrm{PAH}$ population, albeit generally fairly minor ones. The nature of the change is dependent on the placement of the multiple $\mathrm{D}$ atoms.

If one assumes that the placement of D atoms on interstellar PAHs is random, i.e., that there are no favored molecular edge sites for the deuterium, then most multiple deuterations involving only a few D atoms will involve configurations in which the $\mathrm{D}$ atoms all reside on separate rings. For example, in 10 of the 11 deuterium distribution configurations of the doubly deuterated PAH coronene $\left(\mathrm{C}_{24} \mathrm{H}_{10} \mathrm{D}_{2}\right)$, the two $\mathrm{D}$ atoms reside on separate rings. In these cases there will be very little interaction between the different $\mathrm{D}$ atoms and the principal result will simply be to enhance, by the corresponding enrichment factor, the various spectral effects described in the earlier sections of this paper.

The spectral changes resulting from multiple D atoms lying on the same ring are somewhat more complex. If there are enough $\mathrm{D}$ atoms to fully deuterate a ring, then the ring will behave much as it would in a perdeuterated (all $\mathrm{H}$ replaced by
D) version of the PAH. Descriptions of the spectra of a number of perdeuterated PAHs containing a variety of adjacency groups can be found in Bauschlicher et al. (1997). If the number of $\mathrm{D}$ atoms on a ring are insufficient to fully deuterate the ring, then one will see the same sorts of spectral alterations as those associated with adjacency group changes shown in Figure 7. For example, random placement of two D atoms on a trio ring would result in two-thirds of the rings having one solo- $\mathrm{CH}$ and one duet-CD group, while one-third of the rings would have one solo- $\mathrm{CH}$ and two solo-CD groups. Each of these variants would produce a different infrared spectrum that, to first order, would look like the proportional mixing of the relevant functional groups/combinations present.

Again, while such effects can add a great deal of complexity to the spectra of individual PAH variants, they are likely to have a relatively minor effect on the spectrum of the overall population, since most PAHs will be undeuterated and of those that do carry deuterium, only a minor fraction are likely to carry multiple, adjacent $\mathrm{D}$ atoms. Thus, we have not attempted to calculate the much more extensive number of molecular combinations that involve two or more D atoms on the same $\mathrm{PAH}$, although such calculations could be carried out if future telescopic observations should demonstrate that multiple deuteration of PAHs is unexpectedly significant.

\section{CONCLUSIONS}

PAHs are expected to be significantly enriched in D in many different interstellar environments. In most cases this enrichment will be manifested either by (1) the replacement of a single peripheral $\mathrm{H}$ atom with a $\mathrm{D}$ atom, or by (2) the addition of a D atom to an peripheral ring that is converted to aliphatic bonding. Since the degree and distribution of the enrichment depend on the PAHs' chemical history and the specific enrichment processes involved, the detection of dPAHs in space can potentially serve as a powerful tool for understanding chemical processes 
in space. In addition, since many of the organics in primitive meteorites - including aromatic species - are highly enriched in D, dPAHs may serve as excellent tracers for understanding how interstellar materials are incorporated into forming stellar systems.

Three different types of vibrational modes are affected when a $\mathrm{D}$ atom is substituted for a peripheral $\mathrm{H}$ atom: $\mathrm{C}-\mathrm{H}$ in-plane bending modes, $\mathrm{C}-\mathrm{H}$ out-of-plane bending modes, and $\mathrm{C}-\mathrm{H}$ stretching modes. The fundamental $\mathrm{C}-\mathrm{D}$ vibrational frequency of each of these modes is decreased relative to its $\mathrm{C}-\mathrm{H}$ counterpart. The in-plane bending modes of deuterated PAH ions are moved from 7-9 to 9.5-12 $\mu \mathrm{m}$, where they overlap with neutral PAH modes. The out-of-plane $\mathrm{C}-\mathrm{D}$ bending modes are moved from $11-14$ to $13.5-17.9 \mu \mathrm{m}$, where they partially overlap with $\mathrm{C}-\mathrm{H}$ modes associated with rings having higher $\mathrm{H}$-atom adjacency. The aromatic $\mathrm{C}-\mathrm{H}$ stretching shifts from 3.3 to near $4.4 \mu \mathrm{m}$ when D replaces H. Similarly, the $3.4 \mu \mathrm{m}$ band of the $\mathrm{C}-\mathrm{H}$ stretch in the aliphatic edge rings of $\mathrm{H}_{n}$-PAHs is shifted to near $4.6 \mu \mathrm{m}$ in the $\mathrm{C}-\mathrm{D}$ case. In both cases, the shifted $\mathrm{C}-\mathrm{H}$ stretching modes are shifted to frequencies at which PAHs and $\mathrm{H}_{n}$-PAHs have no other vibrational modes. The degree of the frequency shift for the in-plane and out-of-plane bending modes is somewhat dependent on the structure of the PAH, and the shifts are therefore variable. In contrast, the stretching mode frequencies for both the $\mathrm{C}-\mathrm{H}$ and $\mathrm{C}-\mathrm{D}$ stretches are relatively insensitive to the structure of the PAH. As a secondary effect, the presence of peripheral D atoms on PAHs alters the adjacency statistics of the associated $\mathrm{C}-\mathrm{H}$ modes. This results in some changes in the $\mathrm{C}-\mathrm{H}$ modes themselves, although this should be a minor effect for PAH populations deuterated to the levels expected for most interstellar environments.

Thus, the $\mathrm{C}-\mathrm{D}$ stretching modes in the 4.4-4.6 $\mu \mathrm{m}$ region offer the best prospects for observing features that can be unambiguously attributed to deuterated PAHs and related species in interstellar emission spectra. All the remaining $\mathrm{C}-\mathrm{D}-$ involved modes of these species fall in regions of the midinfrared that are also populated to a greater or lesser extent by modes of undeuterated species. Nonetheless, these other modes can still potentially serve as checks for consistency with any $\mathrm{C}-\mathrm{D}$ stretching mode detections. Since the $\mathrm{C}-\mathrm{D}$ stretching modes fall in a wavelength region obscured by telluric $\mathrm{CO}_{2}$ absorption (as do the $\mathrm{C}-\mathrm{D}$ out-of-plane bending mode bands), a proper study of the degree of deuteration of interstellar PAHs will require the use of space-based spectrometers that have spectroscopic capabilities in the appropriate wavelength regions.

The authors gratefully acknowledge support for this work from the NASA Laboratory Astrophysics (188-02-04-02), Long Term Space Astrophysics (399-20-61), and Origins of Solar Systems (344-37-44-01) programs. We also would like to thank E. Peeters, L. Allamandola, and A. Tielens for useful discussions during the preparation of this manuscript and an anonymous referee for his/ her helpful comments and suggestions.

\section{REFERENCES}

Aikawa, Y., \& Herbst, E. 1999, ApJ, 526, 314

Allamandola, L. J., Hudgins, D. M., \& Sandford, S. A. 1999, ApJ, 511, L115 Allamandola, L. J., Tielens, A. G. G. M., \& Barker, J. R. 1989, ApJS, 71, 733 Bauschlicher, C. W., \& Langhoff, S. R. 1997, Spectrochim. Acta A, 53, 1225 Bauschlicher, C. W., Jr., Langhoff, S. R., Sandford, S. A., \& Hudgins, D. M. 1997, J. Phys. Chem. A, 101, 2414

Becke, A. D. 1993, J. Chem. Phys., 98, 5648

Bellamy, L. J. 1958, The Infrared Spectra of Complex Organic Molecules (New York: Wiley)

Bernatowicz, T. J., \& Zinner, E., eds. 1997, AIP Conf. Proc. 402, Astrophysical Implications of the Laboratory Study of Presolar Materials (Woodbury: AIP)

Bernstein, M. P., Dworkin, J., Sandford, S. A., \& Allamandola, L. J. 2001, Meteoritics Planet. Sci., 36, 351

Bernstein, M. P., Sandford, S. A., \& Allamandola, L. J. 1996, ApJ, 472, L127 . 2000, ApJ, 542, 894

Bernstein, M. P., Sandford, S. A., Allamandola, L. J., \& Chang, S. 1994, J. Phys. Chem., 98, 12206

Bernstein, M. P., Sandford, S. A., Allamandola, L. J., Chang, S., \& Scharberg, M. A. 1995, ApJ, 454, 327

Bernstein, M. P., Sandford, S. A., Allamandola, L. J., Gillette, J. S., Clemett, S. J., \& Zare, R. N. 1999, Science, 283, 1135

Bregman, J. D., Hayward, T. L., \& Sloan, G. C. 2000, ApJ, 544, L75

Brooke, T. Y., Sellgren, K., \& Geballe, T. R. 1999, ApJ, 517, 883

Brooke, T. Y., Sellgren, K., \& Smith, R. G. 1996, ApJ, 459, 209

Brown, P. D., \& Charnley, S. B. 1990, MNRAS, 244, 432

Charnley, S. B., Tielens, A. G. G. M., \& Rodgers, S. D. 1997, ApJ, 482, L203 Chiar, J. E., Tielens, A. G. G. M., Whittet, D. C. B., Schutte, W. A., Boogert, A. C. A., Lutz, D., van Dishoeck, E. F., \& Bernstein, M. P. 2000, ApJ, 537, 749 Cronin, J. R., Pizzarello, S., Epstein, S., \& Krishnamurthy, R. V. 1993, Geochim. Cosmochim. Acta, 57, 4745

Dalgarno, A., \& Lepp, S. 1984, ApJ, 287, L47

d'Hendecourt, L. B., Allamandola, L. J., \& Greenberg, J. M. 1985, A\&A, 152,130

Epstein, R. I., Lattimer, J. M., \& Schramm, D. N. 1976, Nature, 263, 198

Frisch, M. J., Pople, J. A., \& Binkley, J. S. 1984, J. Chem. Phys., 80, 3265

Frisch, M. J., et al. 1998, Gaussian 98 (rev. A.9; Pittsburgh: Gaussian, Inc.)

Geiss, J., \& Reeves, H. 1981, A\&A, 93, 189

Goto, M., et al. 2003, ApJ, 589, 419

Hasegawa, T. I., Herbst, E., \& Leung, C. M. 1992, ApJS, 82, 167

Herbst, E., Adams, N. G., Smith, D., \& Defrees, D. J. 1987, ApJ, 312, 351
Hony, S., Van Kerckhoven, C., Peeters, E., Telens, A. G. G. M., Hudgins, D. M., \& Allamandola, L. J. 2001, A\&A, 370, 1030

Hudgins, D. M., \& Allamandola, L. J. 1999a, ApJ, 513, L69 1999b, ApJ, 516, L41

Hudgins, D. M., Bauschlicher, C. W., \& Allamandola, L. J. 2001, Spectrochim. Acta A, 57, 907

Jacq, T., Walmsley, C. M., Henkel, C., Baudry, A., Mauersberger, R., \& Jewell, P. R. 1990, A\&A, 228, 447

Jedamzik, K., \& Fuller, G. M. 1997, ApJ, 483, 560

Kerridge, J. F., \& Chang, S. 1985, in Protostars and Planets II, ed. D. C. Black \& M. S. Matthews (Tucson: Univ. Arizona Press), 738

Kerridge, J. F., Chang, S., \& Shipp, R. 1987, Geochim. Cosmochim. Acta, 51,2527

Langhoff, S. R. 1996, J. Phys. Chem., 100, 2819

Loinard, L., Castets, A., Ceccarelli, C., Tielens, A. G. G. M., Faure, A., Caux, E., \& Duvert, G. 2000, A\&A, 359, 1169

Mattioda, A. L., Hudgins, D. M., Bauschlicher, C. W., Rosi, M., \& Allamandola, L. J. 2003, J. Phys. Chem. A, 107, 1486

Mauersberger, R., Henkel, C., Jacq, T., \& Walmsley, C. M. 1988, A\&A, 194, L1

Messenger, S. 2000, Nature, 404, 968

Millar, T. J., Bennett, A., \& Herbst, E. 1989, ApJ, 340, 906

Millar, T. J., Roberts, H., Markwick, A. J., \& Charnley, S. B. 2000, Philos. Trans. R. Soc. London A, 358, 2535

O’Meara, J. M., Tytler, D., Kirkman, D., Suzuki, N., Prochaska, J. X., Lubin, D., \& Wolfe, A. M. 2001, ApJ, 552, 718

Pagel, B. E. J. 1997, Nucleosynthesis and Chemical Evolution of Galaxies (Cambridge: Cambridge Univ. Press)

Parise, B., et al. 2002, A\&A, 393, L49

Peeters, E., Allamandola, L. J., Bauschlicher, C. W., Jr., Hudgins, D. M., Sandford, S. A., \& Tielens, A. G. G. M. 2004, ApJ, 604, 252

Peeters, E., Hony, S., Van Kerkhoven, C., Tielens, A. G. G. M., Allamandola, L. J., Hudgins, D. M., \& Bauschlicher, C. W. 2002, A\&A, 390, 1089

Reeves, H., Audouze, J., Fowler, W., \& Schramm, D. N. 1973, ApJ, 179, 909

Roueff, E., Tiné, S., Coudert, L. H., Pineau des Forêts, G., Falgarone, E., \& Gerin, M. 2000, A\&A, 354, L63

Sandford, S. A. 1991, ApJ, 376, 599

. 2002, Planet. Space Sci., 50, 1145

Sandford, S. A., \& Allamandola, L. J. 1993, ApJ, 417, 815

Sandford, S. A., Bernstein, M. P., \& Allamandola, L. J. 2004, ApJ, 607, 346 
Sandford, S. A., Bernstein, M. P., Allamandola, L. J., Gillette, J. S., \& Zare, R. N. 2000, ApJ, 538, 691

Sandford, S. A., Bernstein, M. P., \& Dworkin, J. P. 2001, Meteoritics Planet. Sci., 36, 1117

Sellgren, K., Brooke, T. Y., Smith, R. G., \& Geballe, T. R. 1995, ApJ, 449, L69

Stephens, P. J., Devlin, F. J., Chabalowski, C. F., \& Frisch, M. J. 1994, J. Phys. Chem., 98, 11623

Tielens, A. G. G. M. 1983, A\&A, 119, 177

1992, in IAU Symp. 150, Astrochemistry of Cosmic Phenomena, ed. P. D. Singh (Dordrecht: Kluwer), 91

1997, in AIP Conf. Proc. 402, Astrophysical Implications of the Laboratory Study of Presolar Materials, ed. T. J. Bernatowicz \& E. K. Zinner (Woodbury: AIP), 523
Tielens, A. G. G. M., \& Hagen, W. 1982, A\&A, 114, 245

Turner, B. E. 2001, ApJS, 136, 579

Tytler, D., Fan, X. M., \& Burles, S. 1996, Nature, 381, 207

van der Tak, F. F. S., Schilke, P., Müller, H. S. P., Lis, D. C., Phillips, T. G., Gerin, M., \& Roueff, E. 2002, A\&A, 388, L53

Van Kerckhoven, C., et al. 2000, A\&A, 357, 1013

Vastel, C., Phillips, T. G., Ceccarelli, C., \& Pearson, J. 2003, ApJ, 593, L97

Vastel, C., Phillips, T. G., \& Yoshida, H. 2004, ApJ, 606, L127

Vidal-Madjar, A., et al. 1998, A\&A, 338, 694

Zinner, E. 1997, in AIP Conf. Proc. 402, Astrophysical Implications of the Laboratory Study of Presolar Materials, ed. T. J. Bernatowicz \& E. Zinner (Woodbury: AIP), 3 